\title{
Editorial
}

\section{Elevated Troponin Levels in Stable Patients Undergoing Hemodialysis: A Red Flag or a Red Herring?}

\author{
Muthiah Vaduganathan Deepak L. Bhatt \\ Brigham and Women's Hospital Heart and Vascular Center and Harvard Medical School, Boston, Mass., USA
}

Cardiac troponin measurements are central to the prompt diagnosis and management of patients presenting with acute coronary syndromes. These regulatory proteins are sensitive, stable, and reproducible markers of myocardial injury. The widespread utilization of cardiac troponins and their detection using higher-sensitivity assays have identified a subset of stable, asymptomatic patients with elevated troponin levels. Abnormal troponin assays are perhaps most frequently encountered in patients with end-stage renal disease (ESRD) undergoing hemodialysis (HD). Indeed, new-generation, high-sensitivity troponin- $\mathrm{T}$ is elevated in almost all patients, and troponin-I in up to $30 \%$ of patients undergoing HD [1]. A recent meta-analysis of 98 small studies demonstrated that elevated troponin-T and troponin-I levels in patients with chronic kidney disease were robustly associated with increased risk for all-cause and cardiovascular mortality, regardless of requirement for HD [2]. Serial sampling of troponins may optimize the diagnostic yield, test characteristics, and predictive value of these biomarkers when compared with static measurement [3]. Elevated troponins are a frequent source of clinical uncertainty in this population, and as such, we summarize emerging data to

\section{KARGER}

E-Mail karger@karger.com

www.karger.com/ajn inform the pathogenesis, clinical implications, and potential therapeutic applications of elevated troponins in stable patients with ESRD undergoing HD.

\section{Troponin Elevation in ESRD - Flying Under the Radar}

The pathogenesis of this observed subclinical cardiac injury pattern has not been firmly elucidated, but reduced renal clearance is thought to contribute to the high prevalence of circulating, low-level troponin levels in ESRD. However, contrary to prevailing belief regarding HD-mediated biomarker clearance, troponins are increased immediately after standard HD sessions, perhaps related to hemoconcentration [4]. Mechanisms underlying troponin release that have been posited include (1) direct myocardial damage from uremia, circulating endotoxins, and an adverse metabolic milieu; (2) subclinical epicardial or microvascular coronary artery disease (CAD) and heart

Reference article: High ultrafiltration rates increase troponin levels in stable hemodialysis patients.
(2) 2016 S. Karger AG, Basel

0250-8095/16/0433-0170\$39.50/0 
failure; and (3) 'demand' ischemia secondary to volume shifts and hemodynamic stressors during HD.

In this issue of the American Journal of Nephrology, Mavrakanas et al. [5] tackle the influence of HD-specific factors on serial troponin levels in clinically stable patients with ESRD. In this observational cohort study, the investigators profiled troponin-I levels in 236 stable, asymptomatic patients receiving chronic, intermittent $\mathrm{HD}$ at 2 tertiary care centers. Almost a quarter of patients had at least one abnormal pre-HD troponin-I level over the 3 consecutive months of measurement. Troponin elevations were mild (mostly less than $0.20 \mu \mathrm{g} / \mathrm{l}$ ) and a majority were transient. In a linear mixed-effects model, high ultrafiltration rates were independently associated with abnormal troponin measurements [5].

Despite the inherent limitations of their observational study design and small sample size, Mavrakanas et al. [5] advance our understanding of the natural history and biological variability of this commonly measured biomarker to a great extent. Cardiac troponins were above the 99th percentile for a healthy population in a substantial proportion of ESRD patients, even with conservative assays and in an apparently clinically stable cohort with a low-prevalence of established CAD ( 30\%). Measured troponin levels fluctuated in many patients near the assay cut-off, and were importantly related to certain HD-related factors.

\section{Detection of Troponin in a Stable Patient - An Opportunity for Intervention?}

Multivessel CAD is frequently observed in angiographic studies of asymptomatic HD patients [6], and rates of cardiovascular disease are expected to rise with the aging population and associated metabolic comorbidities. Despite the high burden of CAD and its attendant adverse health and economic impact in patients with ESRD on HD, definitive tools to characterize individual patient risk and strategies to ameliorate this event burden remain elusive.

Elevated troponins may serve as an important adjunct in the clinical care of ESRD, potentially identifying patients who may benefit from more aggressive cardiovascular evaluation and therapeutic intervention. In fact, the routine measurement of troponin in stable ESRD patients is given some degree of support by recent clinical practice guidelines for cardiovascular evaluation prior to kidney transplantation (class IIb; level of evidence B) [7].

Troponins in ESRD
Although an aggressive therapeutic approach to ESRD patients with abnormal cardiac biomarkers is intuitively appealing, limited data are available to guide clinical decision making. Routine coronary angiography has not been rigorously studied [8] and may have a limited role in this population, given drivers of ischemia other than epicardial CAD that would be expected to be less responsive to revascularization. There is also potential for harm in the context of increased site-related bleeding complications and further reduction of residual renal function. In a recent analysis from the BARI 2D (Bypass Angioplasty Revascularization Investigation in Type 2 Diabetes; ClinicalTrials.gov Identifier: NCT00006305) trial, nearly $40 \%$ of patients with type 2 diabetes and stable CAD had abnormal baseline troponin- $\mathrm{T}$ concentrations using a sensitive assay [9]. In this subset with elevated baseline troponin-T levels, randomization to early revascularization did not alter subsequent risk of clinical events or serial troponin trajectory [9]. Based on work by Mavrakanas et al. [5] and others, optimizing hemodynamics and limiting cardiovascular stressors during HD may attenuate repeated subclinical cardiac injury. Increasing the frequency of HD has been proposed to reduce hemodynamic perturbations, alterations in left ventricular function, and troponin release compared to the outcomes of conventional HD [10], but this approach requires validation in large prospective randomized studies.

The diagnosis of CAD in ESRD is challenging due to atypical presentations in the context of comorbid diabetes mellitus, autonomic dysfunction, and exercise intolerance, and limited reliability and sensitivity of traditional noninvasive testing. At the present time, the detection of abnormal cardiac biomarkers in otherwise stable patients may prompt the intensification of medical therapies directed toward CAD in this high-risk cohort. HD presents unique recurrent hemodynamic stresses to the cardiovascular system, and pending further research, troponin release in otherwise asymptomatic ESRD patients may represent an opportunity to ameliorate unacceptably high rates of cardiovascular morbidity and mortality in this population.

\section{Disclosure Statement}

Dr. M. Vaduganathan has no relevant disclosures related to this work. Dr. D.L. Bhatt discloses the following relationships - Advisory Board: Cardax, Elsevier Practice Update Cardiology, Medscape Cardiology, Regado Biosciences; Board of Directors: Boston VA Research Institute, Society of Cardiovascular Patient Care; Chair: American Heart Association Get with the Guidelines Steering Committee; Data Monitoring Committees: Duke Clinical Re-

Am J Nephrol 2016;43:170-172

DOI: $10.1159 / 000445363$ 
search Institute, Harvard Clinical Research Institute, Mayo Clinic, Population Health Research Institute; Honoraria: American College of Cardiology (Senior Associate Editor, Clinical Trials and News, ACC.org), Belvoir Publications (Editor in Chief, Harvard Heart Letter), Duke Clinical Research Institute (Clinical Trial Steering Committees), Harvard Clinical Research Institute (Clinical Trial Steering Committee), HMP Communications (Editor in Chief, Journal of Invasive Cardiology), Journal of the American College of Cardiology (Guest Editor; Associate Editor), Population
Health Research Institute (Clinical Trial Steering Committee), Slack Publications (Chief Medical Editor, Cardiology Today's Intervention), WebMD (CME Steering Committees); Other: Clinical Cardiology (Deputy Editor); Research Funding: Amarin, AstraZeneca, Bristol-Myers Squibb, Eisai, Ethicon, Forest Laboratories, Ischemix, Medtronic, Pfizer, Roche, Sanofi Aventis, The Medicines Company; Site Co-Investigator: Biotronik, Boston Scientific, St. Jude Medical; Trustee: American College of Cardiology; Unfunded Research: FlowCo, PLx Pharma, Takeda.

\section{References}

1 Mbagaya W, Luvai A, Lopez B: Biological variation of cardiac troponin in stable haemodialysis patients. Ann Clin Biochem 2015; 52(pt 5):562-568.

2 Michos ED, Wilson LM, Yeh HC, Berger Z, Suarez-Cuervo C, Stacy SR, Bass EB: Prognostic value of cardiac troponin in patients with chronic kidney disease without suspected acute coronary syndrome: a systematic review and meta-analysis. Ann Intern Med 2014;161: 491-501.

3 Jacobs LH, van de Kerkhof J, Mingels AM, Kleijnen VW, van der Sande FM, Wodzig WK, Kooman JP, van Dieijen-Visser MP: Haemodialysis patients longitudinally assessed by highly sensitive cardiac troponin $\mathrm{T}$ and commercial cardiac troponin $\mathrm{T}$ and cardiac troponin I assays. Ann Clin Biochem 2009;46(pt 4):283-290.
4 Conway B, McLaughlin M, Sharpe P, Harty J: Use of cardiac troponin $\mathrm{T}$ in diagnosis and prognosis of cardiac events in patients on chronic haemodialysis. Nephrol Dial Transplant 2005;20:2759-2764.

5 Mavrakanas TA, Sniderman AD, Barré PE, Vasilevsky M, Alam A: High ultrafiltration rates increase troponin levels in stable hemodialysis patients. Am J Nephrol 2016;43:173-178.

6 Charytan D, Kuntz RE, Mauri L, DeFilippi C: Distribution of coronary artery disease and relation to mortality in asymptomatic hemodialysis patients. Am J Kidney Dis 2007;49:409-416.

7 Lentine KL, Costa SP, Weir MR, Robb JF, Fleisher LA, Kasiske BL, Carithers RL, Ragosta M, Bolton K, Auerbach AD, Eagle KA: Cardiac disease evaluation and management among kidney and liver transplantation candidates: a scientific statement from the
American Heart Association and the American College of Cardiology Foundation: endorsed by the American Society of Transplant Surgeons, American Society of Transplantation, and National Kidney Foundation. Circulation 2012;126:617-663.

8 Manske CL, Wang Y, Rector T, Wilson RF, White CW: Coronary revascularisation in insulin-dependent diabetic patients with chronic renal failure. Lancet 1992;340:998-1002.

9 Everett BM, Brooks MM, Vlachos HE, Chaitman BR, Frye RL, Bhatt DL: Troponin and cardiac events in stable ischemic heart disease and diabetes. N Engl J Med 2015;373:610-620.

10 Jefferies HJ, Virk B, Schiller B, Moran J, McIntyre CW: Frequent hemodialysis schedules are associated with reduced levels of dialysisinduced cardiac injury (myocardial stunning). Clin J Am Soc Nephrol 2011;6:1326-1332. 